\title{
Relationship between Sagittal Alignment and Anterior Bony Resorption of Cervical Vertebral Body in Patients with Ankylosing Spondylitis
}

\author{
Ki-Tack Kim ${ }^{1}$, Kee-Yong $\mathrm{Ha}^{1}$, Yong-Chan $\mathrm{Kim}^{1}$, Keun-Ho Lee ${ }^{2}$, \\ Sang-Il Kim ${ }^{3}$, Young-Hoon $\mathrm{Kim}^{3}$, Sung-Min Kim ${ }^{1}$

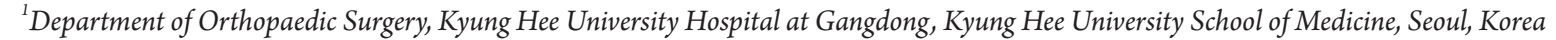 \\ ${ }^{2}$ Department of Orthopaedic Surgery, Hallym University Kangdong Sacred Heart Hospital, College of Medicine, Hallym University, Seoul, Korea \\ ${ }^{3}$ Department of Orthopaedic Surgery, Seoul St. Mary's Hospital, College of Medicine, The Catholic University of Korea, Seoul, Korea
}

Study Design: This is a retrospective comparative cohort study.

Purpose: To investigate the prevalence of anterior resorption of the cervical spine in ankylosing spondylitis (AS) and its relationship with sagittal alignment.

Overview of Literature: This study believes that no published reports exist regarding the relationship between anterior resorption and spinopelvic alignment.

Methods: This retrospective study included 238 patients with AS who underwent surgical correction for thoracolumbar kyphosis. Of these, 80 patients with complete subaxial ankylosis were selected and divided into two groups: groups 1 (consisting of 28 patients with anterior bony resorption) and 2 (consisting of 52 patients without anterior resorption). Cervical sagittal parameters were analyzed and compared between the two groups.

Results: The average lateral diameter measured in patients with C5 anterior resorption was $84.2 \% \pm 7.8 \%(62.4 \%-96.8 \%)$. Cervical lordosis was $8.7^{\circ} \pm 13.4^{\circ}$ and $10.9^{\circ} \pm 11.5^{\circ}$ in groups 1 and $2(p=0.556)$, respectively. No significant differences were noted between the two groups on spinopelvic alignment in the $\mathrm{T} 1$ slope $\left(52.2^{\circ} \pm 11.1^{\circ}\right.$ and $53.3^{\circ} \pm 9.9^{\circ}$ in groups 1 and 2 , respectively; $\left.p=0.742\right), \mathrm{C} 2-$ C7 sagittal vertical axis (SVA; $6.2 \pm 1.7 \mathrm{~cm}$ and $6.2 \pm 1.8 \mathrm{~cm}$ in groups 1 and 2, respectively; $p=0.978), C 7$ SVA $(14.3 \pm 4.9 \mathrm{~cm}$ and $14.6 \pm 6.2$ $\mathrm{cm}$ in groups 1 and 2 , respectively; $p=0.823)$, or T1 pelvic angle $\left(27.1^{\circ} \pm 8.9^{\circ}\right.$ and $31.6^{\circ} \pm 11.2^{\circ}$ in groups 1 and 2 , respectively; $\left.p=0.382\right)$. Correlation analyses were significant between the extent of anterior resorption and sagittal parameters, $C 2-C 7$ lordosis $\left(R^{2}=-0.428\right.$, $p=0.021)$, and T1-T4 kyphosis ( $\left.R^{2}=-0.375, p=0.045\right)$.

Conclusions: Anterior bony resorption could develop by stress concentration. However, the development was not related to the sagittal alignment. The particular segments involved in developing anterior resorption varied, possibly because of their dependence on the preceding pattern of ankylosis.

Keywords: Anterior resorption; Cervical vertebrae; Ankylosing spondylitis; Sagittal alignment

Received Dec 27, 2020; Revised Mar 31, 2021; Accepted Apr 18, 2021

Corresponding author: Kee-Yong Ha

Department of Orthopaedic Surgery, Kyung Hee University Hospital at Gangdong, Kyung Hee University School of Medicine, 892 Dongnam-ro, Gangdong-gu, Seoul 05278, Korea

Tel: +82-2-440-7497, Fax:+82-2-440-7498, E-mail: kyh@catholic.ac.kr 


\section{Introduction}

Several different radiological findings in ankylosing spondylitis (AS) exist, with different pathogenesis in the cervical spine (e.g., squaring, syndesmophytes, ankylosis, vertebral body erosion, craniocervical junction involvement, and ossification of the longitudinal ligaments) [18]. Adaptive remodeling of the deformed cervical spine is well observed in patients with AS with severe thoracolumbar kyphosis. The change in mechanical loading may be one control stimulus to enhance the remodeling activities involving mechanisms (e.g., bone growth, absorption, and reinforcement) $[9,10]$. Superficial erosions of the anterior surfaces of the cervical vertebral bodies, so-called "anterior spondylitis", are known in AS and account for the characteristic "squaring" of the vertebral bodies [11-13]. However, these superficial erosions seem to be a late manifestation associated with complete ankylosis. Moreover, extreme kyphosis of the thoracolumbar spine is when the cervicothoracic segments tend to be horizontal with the patient in the upright position to obtain a horizontal gaze. Probably as a result of this continual stress, physiological resorption of bone occurs at the anterior, convex surface of the cervical arch by Wolff's law of transformation [14]. Thus, this effect is probably superimposed on superficial erosions in this region, thereby accounting for the degree of bone loss.

Not all patients with complete ankylosis of the cervical spine show anterior bony resorption or erosion of the cervical vertebral body even in patients with AS with severe kyphotic deformity of the thoracolumbar spine. Important issues remain to be addressed, even though the pathogenesis of anterior resorption of the cervical vertebra in patients with AS could be defined clearly. First, the prevalence of anterior resorption of the cervical vertebral bodies in patients with AS is unknown, and it is believed that it has not been estimated in any previous reports. Second, determining whether anterior resorption is related to the sagittal spinopelvic alignment is essential. Therefore, this study aimed to investigate the prevalence of anterior bony resorption of the cervical vertebral bodies and to evaluate the relationship between anterior resorption of the cervical spine and spinopelvic alignment in patients with AS with severe thoracolumbar kyphosis.

\section{Materials and Methods}

\section{Patient selection}

This study was approved by the Institutional Review Board of Kyung Hee University Hospital at Gangdong before data collection (IRB no., 2020-03-023) and informed consent was waived. This study retrospectively reviewed 238 patients with AS who underwent surgical correction for severe thoracolumbar kyphosis from January 2012 to December 2019. The states of ankylosis of the whole spines and both sacroiliac joints were confirmed by whole-body and pelvic plain radiographs and computed tomography (CT) imaging. In addition, the states of cervical ankylosis in all patients were analyzed using the modified Stoke Ankylosing Spondylitis Spinal Score (mSASSS) $[15,16]$.

\section{Cervical spine}

The lower border of the $\mathrm{C} 2$ up to and including the upper border of the $\mathrm{T} 1$ was viewed on the lateral radiograph. The nominal scoring system was used $(0=$ no abnormality; $1=$ erosion, sclerosis, or squaring; $2=$ syndesmophyte; and $3=$ total bony bridging at each site). Cases were excluded to reduce patient selection bias if the confirmation of ankylosis of the cervical spine was doubtful. An experienced spine surgeon who did not participate in the surgery scored the mSASSS.

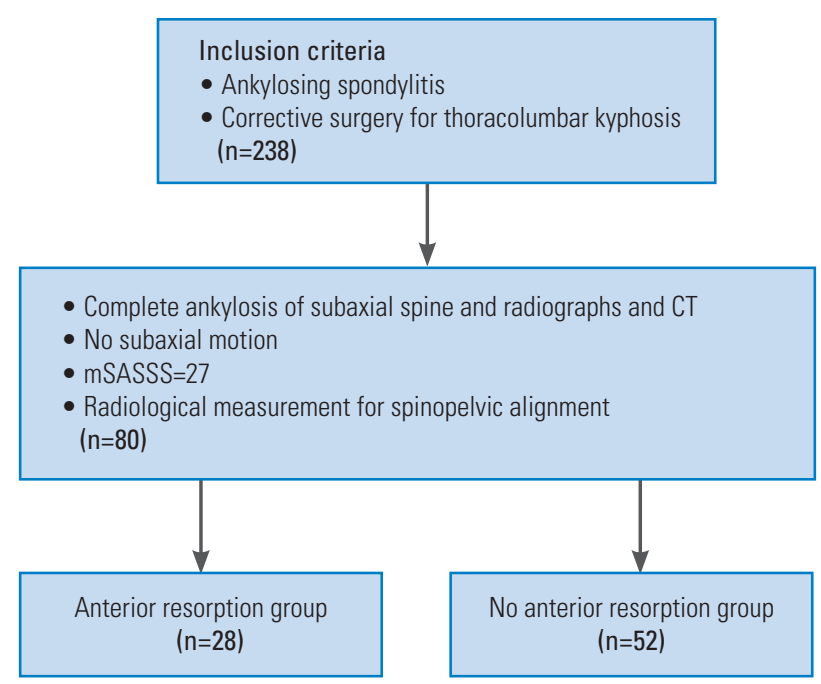

Fig. 1. Study design. CT, computed tomography; mSASSS, modified Stoke Ankylosing Spondylitis Spinal Score. 
The inclusion criteria in this study were (1) the presence of subaxial cervical spine with complete ankylosis (total bony bridging) on the plain radiographs and CT, (2) the absence of subaxial motion with or without craniocervical involvement confirmed by dynamic flexion-extension cervical radiographs, and (3) a mSASSS of 36, indicating complete subaxial ankylosis. Among the 238 patients identified, 80 patients who met the study criteria were enrolled in this study and divided into two groups according to the presence or absence of anterior bony resorption of the cervical bodies (Fig. 1).

\section{Radiological assessments}

The following spinal parameters were assessed on the lateral radiographs: (1) C2-C7 lordosis angle; (2) C2-C7 sagittal vertical axis (SVA); (3) C0-C7 lordosis angle (between the McGregor line and the lower C2 endplate); (4) T1 slope (between the upper T1 endplate and the horizontal line); (5) C7 SVA, which was measured as the distance between the C7 plumb line and the S1 posterosuperior corner; (6) T1 pelvic angle (T1PA), which was measured as the angle between the line from the femoral head axis

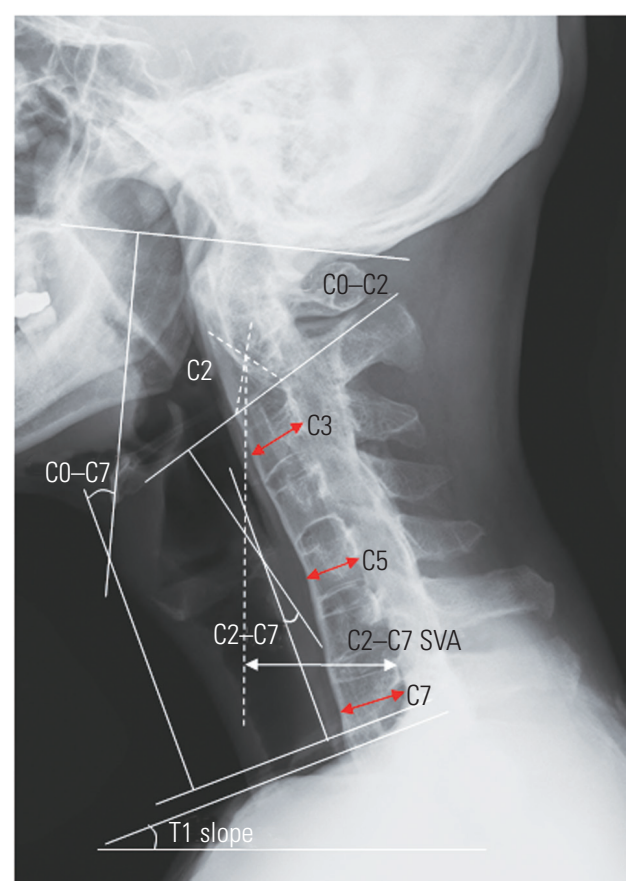

Fig. 2. Radiographic measurements of the cervical spine: C2-C7 lordosis angle, C2-C7 sagittal vertical axis (C2-C7 SVA), CO-C7 lordosis angle, and T1 slope. The percentage of anterior resorption of vertebral body was obtained by ratio of the narrowest anteroposterior distance of scaffolding vertebra to the mean anteroposterior distance of the two adjacent cervical vertebral bodies. to the $\mathrm{T} 1$ centroid and the line from the femoral head axis to the middle of the S1 superior endplate; (7) T1-T4 kyphosis, which was measured from the upper T1 endplate to the lower T4 endplate; and (8) T4-T12 kyphosis, which was measured from the upper T4 endplate to the lower T12 endplate (Fig. 2). The percentage of anterior resorption at each segment was calculated by the ratio between the narrowest anteroposterior distance of the scaffolding vertebra and the mean anteroposterior distances of the two vertebral bodies above and below (Fig. 2).

\section{Statistical analyses}

An independent $t$-test and chi-square test were used to compare the two groups. The Spearman correlation analysis was performed to determine the association between the extent of anterior resorption and radiographic parameters. Statistical analyses were performed using IBM SPSS Statistics for Windows software ver. 22.0 (IBM Corp., Armonk, NY, USA), and $p$-values $<0.05$ were considered statistically significant.

\section{Results}

\section{Patient data and prevalence of anterior resorption}

Of the patients, 238 underwent surgical correction for thoracolumbar kyphosis. Consequently, 80 (33.6\%) of the 238 patients showed complete ankylosis of the cervical spine without any subaxial motions. The patients were five women and 75 men, with a mean age of $47.2 \pm 9.7$ years. Furthermore, the 80 patients showing complete ankylosis of the cervical spine were divided into two groups according to anterior resorption of the cervical vertebral bodies. Thus, 28 patients showed anterior resorption of the cervical vertebral bodies (group 1) (Fig. 3) and 52 patients did not show anterior resorption of the cervical vertebral bodies (group 2) (Fig. 4). Group 1 was composed of three women and 25 men. The mean age was $46.2 \pm 9.8$ years and $47.8 \pm 10.4$ years for groups 1 and 2, respectively. No statistical differences between the two groups on age and gender ratio were observed (Table 1). The overall prevalence of anterior resorption of the cervical vertebral bodies in all 238 patients was $11.8 \%$. However, of the 80 patients who had complete ankylosis of the cervical vertebra, the overall prevalence of anterior resorption was $35.0 \%$. The disease duration was $21.9 \pm 4.7$ years and $11.9 \pm 2.9$ years in 
groups 1 and 2, respectively, but this was not statistically different between the two groups $(p=0.724)$.

\section{Radiological summary of the patient cohort}

The anterior resorption level of the cervical vertebral bodies developed at C4, C5, and C6 in all patients. The most severe anterior resorption of the cervical vertebral bodies developed at C5 in all patients. The average lateral diameter measurement in patients who had anterior re- sorption at C5 was $84.2 \% \pm 7.8 \%(62.4 \%-90.8 \%)$, which was smaller than that of the adjacent vertebral bodies. Cervical lordosis, measured by the $\mathrm{C} 2-\mathrm{C} 7$ angle, was $8.7^{\circ} \pm 13.4^{\circ}$ and $10.9^{\circ} \pm 11.5^{\circ}$ in groups 1 and 2 , respectively. The between-group difference was insignificant $(p=0.556)$. Moreover, no statistical difference was observed between the two groups on the spinopelvic alignment for the $\mathrm{T} 1$ slope $\left(52.2^{\circ} \pm 11.1^{\circ}\right.$ and $53.3^{\circ} \pm 9.9^{\circ}$ in groups 1 and 2 , respectively; $p=0.742), \mathrm{C} 2-7$ SVA $(6.2 \pm 1.7 \mathrm{~cm}$ and $6.2 \pm 1.8$ $\mathrm{cm}$ in groups 1 and 2, respectively; $p=0.978$ ), C7 SVA
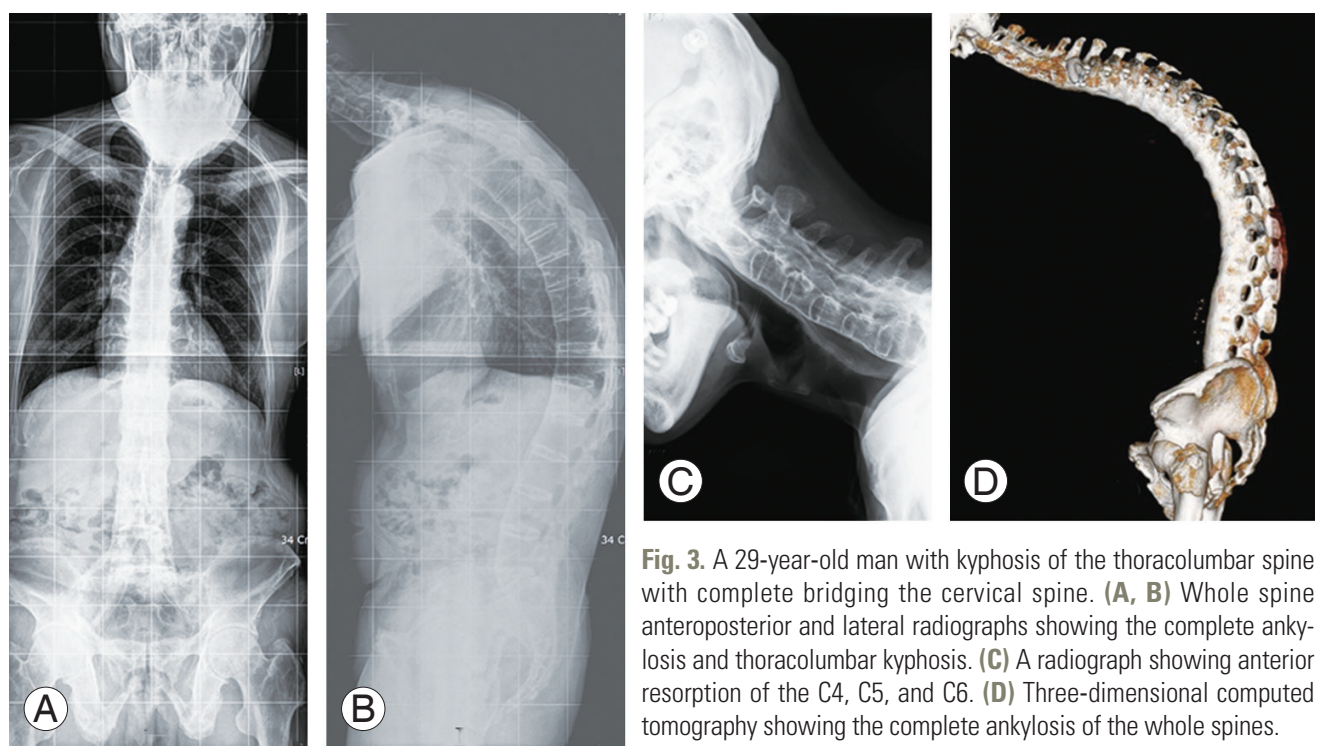

Fig. 3. A 29-year-old man with kyphosis of the thoracolumbar spine with complete bridging the cervical spine. (A, B) Whole spine anteroposterior and lateral radiographs showing the complete ankylosis and thoracolumbar kyphosis. (C) A radiograph showing anterior resorption of the C4, C5, and C6. (D) Three-dimensional computed tomography showing the complete ankylosis of the whole spines.
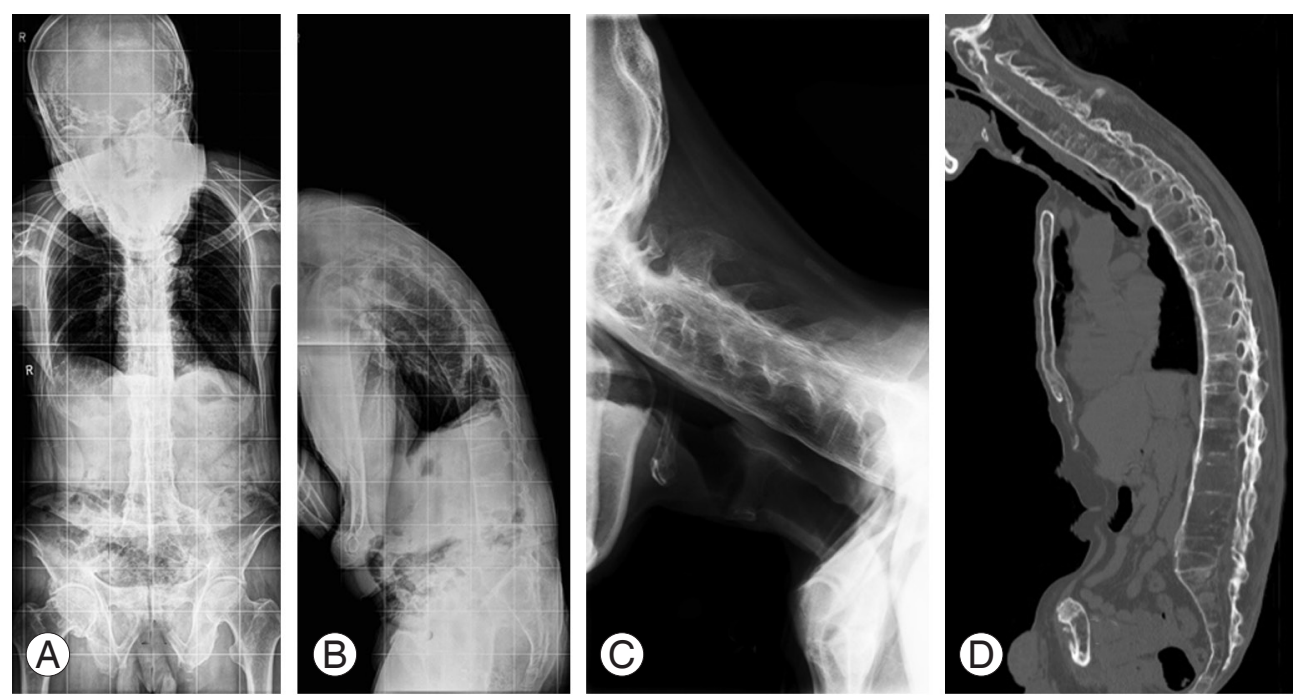

Fig. 4. A 49-year-old man with severe kyphosis of the thoracolumbar spine and complete bridging the cervical spine. (A, B) Whole spine anteroposterior and lateral radiographs. (C) A radiograph showing complete ankylosis of the cervical spine including the occipitocervical junction. No anterior bony resorption of the cervical spines was observed. (D) Computed tomography showing complete ankylosis of the spine without anterior resorption of the cervical spines. 
$(14.3 \pm 4.9 \mathrm{~cm}$ and $14.6 \pm 6.2 \mathrm{~cm}$ in groups 1 and 2 , respectively; $p=0.823)$, and T1PA $\left(27.1^{\circ} \pm 8.9^{\circ}\right.$ and $31.6^{\circ} \pm 11.2^{\circ}$ in groups 1 and 2 , respectively; $p=0.382$ ) (Table 1 ). Correlation analyses between the extent of anterior resorption and radiographic parameters showed significance between the C2-C7 lordosis $\left(R^{2}=-0.428, p=0.021\right)$ and T1-T4 kyphosis $\left(R^{2}=-0.375, p=0.045\right)$ (Table 2$)$.

\section{Craniocervical (CO-C2) involvement}

In the current cohort study of 80 patients, seven patients (8.8\%) showed C0-C2 involvement. Groups 1 and 2 had four patients (14.3\%) and three patients (5.8\%), respectively. All patients were male, with an average age of 52 years (range, 29-62 years). The average age of patients with $\mathrm{C} 0$ C2 involvement was 42 years (range, 29-52 years) and 57.7 years (range, 56-62 years) in groups 1 and 2, respectively. No statistical difference existed between the rates of craniocervical involvement in the two groups $(p=0.232)$.

Table 1. Comparison of demographic and radiographic data

\begin{tabular}{|c|c|c|c|}
\hline Characteristic & Group 1 ( $n=28)$ & Group 2 ( $n=52$ ) & $p$-value \\
\hline Age (yr) & $46.2 \pm 9.8$ & $47.8 \pm 10.4$ & $0.588^{a \mid}$ \\
\hline Sex (female \%) & 10.7 & 9.6 & $1.000^{b)}$ \\
\hline Disease duration (yr) & $21.9 \pm 4.7$ & $11.9 \pm 2.9$ & $0.724^{a \mid}$ \\
\hline CO-C7 lordosis $\left({ }^{\circ}\right)$ & $-35.8 \pm 39.6$ & $-48.3 \pm 22.9$ & $0.169^{a l}$ \\
\hline C2-C7 Iordosis $\left({ }^{\circ}\right)(\mathrm{A})$ & $-8.7 \pm 13.4$ & $-10.9 \pm 11.5$ & $0.556^{a \mid}$ \\
\hline C2-C7 SVA (cm) & $6.2 \pm 1.7$ & $6.2 \pm 1.8$ & $0.978^{\mathrm{al}}$ \\
\hline T1 slope $\left({ }^{\circ}\right)(\mathrm{B})$ & $52.2 \pm 11.1$ & $53.3 \pm 9.9$ & $0.742^{\mathrm{al}}$ \\
\hline$B-A\left({ }^{\circ}\right)$ & $61.0 \pm 21.4$ & $64.2 \pm 18.5$ & $0.589^{a l}$ \\
\hline T1-T4 kyphosis $\left({ }^{\circ}\right)$ & $8.5 \pm 5.8$ & $9.6 \pm 7.8$ & $0.574^{\text {al }}$ \\
\hline T4-T12 kyphosis ( ${ }^{\circ}$ ) & $48.1 \pm 14.9$ & $46.6 \pm 13.2$ & $0.725^{a \mid}$ \\
\hline C7 SVA (cm) & $14.3 \pm 4.9$ & $14.6 \pm 6.2$ & $0.823^{\mathrm{al}}$ \\
\hline T1 pelvic angle $\left(^{\circ}\right)$ & $27.1 \pm 8.9$ & $31.6 \pm 11.2$ & 0.382 \\
\hline
\end{tabular}

Values are presented as mean \pm standard deviation or $\%$.

SVA, sagittal vertical axis.

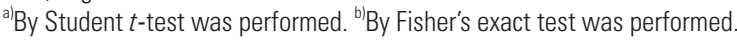

\section{Discussion}

Radiographic changes in the cervical spine have been observed in patients with AS, although the prevalence rates differ among reports [17-19]. Cervical involvement occurs less frequently than lumbosacral AS involvement and at a later time in the course of the illness, because ankylosis usually progresses caudally to cephalad in the cervical spine [17]. Ankylosis can lead to a loss of lumbar spinal lordosis and progressive kyphosis of the cervical and thoracic spine. Cervical involvement increases with age and disease duration in AS and is more frequent in symptomatic and structurally severe forms of the disease [18]. Moreover, cervical spine involvement may progress until the patients are unable to look forward and subsequently show the chin-on-chest deformity [18]. However, the extent and degree of cervical involvement could vary by disease duration because AS progresses over the whole life of the patients and progresses caudally to cephalad [17-19]. One study identified the risk of cervical involvement concerning disease duration and showed that $19.6 \%$, $29.9 \%, 45.1 \%$, and $70.0 \%$ of the patients had radiological involvement after $5,10,15$, and 20 years, respectively [18]. The same group also reported that $21.3 \%$ of the cervical vertebral body eroded, which was similar to the anterior resorption of the vertebral bodies [18]. Furthermore, Lee et al. [2] reported that $48.6 \%$ showed radiological changes to the cervical spine. The average disease duration in the current study was $16.9 \pm 3.9$ years $(21.9 \pm 4.7$ years and $11.9 \pm 2.9$ years in groups 1 and 2, respectively) and $33.6 \%$ of the patients had complete ankylosis of the cervical spine. Of the 80 patients with complete ankylosis, 28 patients $(35 \%)$ showed anterior resorption of the cervical vertebral bodies. However, the current study showed relatively less cervical involvement than any other previous reports $[18,19]$. We believe that this may be because the patients enrolled in this cohort study were selected. After all, they had complete ankylosis of the cervical spine

Table 2. Correlation analysis between the extent of anterior resorption and radiographic parameters

\begin{tabular}{|c|c|c|c|c|c|c|c|c|}
\hline Resorption $\%$ & CO-C7 lordosis & C2-C7 lordosis & C2-C7 SVA & T1 slope & T1-T4 kyphosis & T4-T12 kyphosis & C7 SVA & T1PA \\
\hline$R^{2}$ & -0.305 & $-0.428^{*}$ & -0.282 & 0.163 & $-0.375^{*}$ & 0.212 & -0.097 & -0.295 \\
\hline$p$-value & 0.107 & $0.021^{*}$ & 0.139 & 0.399 & $0.045^{*}$ & 0.269 & 0.617 & 0.490 \\
\hline
\end{tabular}

Spearman test was performed.

SVA, sagittal vertical axis; T1PA, T1 pelvic angle.

*Indicates significant difference $(p<0.05)$. 
without subaxial motion.

Martel et al. $[19,20]$ reported that the cervicothoracic segments tend to be horizontal with the patient in the upright position, which is associated with complete ankylosis and extreme kyphosis of the thoracolumbar spine. Continual neck hyperextension becomes necessary to allow the patient to look forward rather than look down. As a result of this continual stress, physiological bone resorption occurs at the anterior, convex surface of the cervical arch, by Wolff's law [21]. Additionally, the anterior resorption of the cervical vertebral body in AS, a growth disturbance at ring epiphyses, has been reported [2]. Moreover, Abe et al. [22] reported that atrophic changes, similar to anterior resorption, in the cervical vertebral body after cervical fusion in adolescent patients were caused by endplate destruction during anterior interbody fusion (AIF) and instrumentation-induced stress shielding effect. Abe et al. [22] also stressed that the effects of altering load transmission may be greater after posterior spinal fusion with instrumentation than in anterior fusion with instrumentation because the normal alignment of the cervical spine is lordotic. However, this morphological change in AS in the current study occurred without endplate destruction and instrumentation-induced stress shielding effect. In addition, this morphological change can develop in adult patients who undergo AIF of the cervical spine [23]. These radiological findings were similar to the in-waisting sign (a phenomenon of becoming narrow in the waist of the fused vertebral body) of the vertebral body developing after the AIF of the cervical spine [23,24]. Moon et al. [24] also reported the morphological adaptation of bone grafts and fused vertebral bodies after noninstrumented AIF and suggested that the vertebral in-waisting of the surgically fused anterior cervical column indicated maintenance of normal function at the adjacent motion segments. This process is explained by Wolff's law and Frost's flexure drift laws [25-27]. Drift is the basis of bone remodeling. Two kinds of drift, osteoclastic and osteoblastic, remodel bony architecture by moving its periosteal and cortical endosteal surfaces through tissue space, usually traversing to its longitudinal axis [23]. Ha et al. [28] also reported spontaneous osteophyte resorption and sclerosis disappearance at the fused segments by Wolff's law after lumbar fusion instrumentation. Like the in-waisting sign after AIF, the gradual loss of motion at each segment of the spinal column in AS leads to the squaring of the vertebral bodies, which is explained by the flexure drift law [24]. However,
Moon et al. [24] postulated that vertebral bodies are only squared without in-waisting in AS, unlike AIF of the cervical spine. In the current study, 28 of the 80 patients with complete ankylosis of the cervical spine showed anterior bony resorption that was similar to the in-waisting sign after cervical AIF. Interestingly, the anterior resorption of the cervical vertebral bodies developed at $\mathrm{C} 4, \mathrm{C} 5$, and $\mathrm{C} 6$ without the involvement of $\mathrm{C} 3$ and $\mathrm{C} 7$ in all patients. The most severe anterior resorption developed at $\mathrm{C} 5$. The average lateral diameter measured in patients who had anterior resorption of the C5 vertebra was $84.2 \% \pm 7.8 \%$. This could be closely related to the motion of the cervical spine because these segments have larger motion than any other segments in the cervical spine. Moreover, segments that need more motion for horizontal gaze are more likely to develop resorption of the anterior vertebral bodies by Wolff's law. Therefore, more malalignment due to severe kyphosis of the thoracolumbar spine could assume that more anterior resorption may develop because the cervical spine shifts to be more lordotic to allow the patient to obtain a horizontal gaze. Two parameters in the current study were identified as significant (C2-C7 lordosis $\left[R^{2}=-0.428, p=0.021\right]$ and T1-T4 kyphosis $\left[R^{2}=-0.375\right.$, $p=0.045]$ ) in the correlation analyses between the extent of anterior resorption and radiographic parameters. This study hypothesizes that more motion happens to allow for the horizontal gaze and maintain cervical lordosis and more stress concentration at the convex curvature, leading to more resorption by Wolff's law. However, in the current cohort study, no statistical difference was observed between the two groups in terms of spinopelvic alignments including T1 tilt, C2-C7 SVA, thoracic kyphosis, C7 SVA, and T1PA. Therefore, the particular segments involved in developing anterior resorption by Wolff's law varied, possibly because of their dependence on the preceding ankylosis pattern. Slobodin et al. [8] reported that the $\mathrm{C} 0-\mathrm{C} 2$ junction involvement is frequently involved in patients with advanced AS. In the current study, the C0C2 junction involvement was noted in $7(8.8 \%)$ of the 80 patients with cervical involvement. However, no statistical difference was observed between the two groups. In addition, anterior resorption of the vertebral bodies was not affected by ankylosis of the $\mathrm{C} 0-\mathrm{C} 2$ junction.

This study has some limitations. (1) It was a retrospective cohort study with a limited focus on radiological findings. (2) The disease duration could be related to vertebral body resorption, but knowing exactly when AS started in 
most patients is impossible. The notion that cervical involvement increased with disease duration can be debated because it requires accepting the patient's memory as sufficient to determine the onset of disease and cervical spine involvement [18]. Most patients in the current study did not recognize when the initial symptoms started. However, in the current study, patients in group 1 were more likely to pretend to have had a longer disease duration than those in group 2, even though no statistical difference was observed between the two groups concerning disease duration. We believed that this is the first report regarding the evaluation of the prevalence of complete ankylosis of the cervical spine without subaxial motion and pathogenesis of the anterior resorption of the cervical vertebral bodies in patients with AS related to spinopelvic malalignment.

\section{Conclusions}

In the current study, $11.8 \%$ of patients with AS developed anterior resorption of the cervical vertebral bodies. However, anterior resorption may not be related to sagittal malalignment and the severity of the thoracolumbar kyphotic deformity. Therefore, the particular segments involved in developing anterior resorption by Wolff's law varied, possibly because of their dependence on the preceding ankylosis pattern.

\section{Conflict of Interest}

No potential conflict of interest relevant to this article was reported.

\section{Author Contributions}

JYS and KYH designed the study and wrote the manuscript. YHK and HYP analyzed the data. DGC, YHC, and YHR collected the data. SIK revised the manuscript.

\section{References}

1. Reiter MF, Boden SD. Inflammatory disorders of the cervical spine. Spine (Phila Pa 1976) 1998;23:275566.

2. Lee HS, Kim TH, Yun HR, et al. Radiologic changes of cervical spine in ankylosing spondylitis. Clin Rheumatol 2001;20:262-6.

3. Hunter T. The spinal complications of ankylosing spondylitis. Semin Arthritis Rheum 1989;19:172-82.

4. Kahan A, Menkes CJ. Destructive spine lesions in ankylosing spondylitis. Ann Rheum Dis 1990;49:422.

5. Ramos-Remus C, Gomez-Vargas A, Guzman-Guzman JL, et al. Frequency of atlantoaxial subluxation and neurologic involvement in patients with ankylosing spondylitis. J Rheumatol 1995;22:2120-5.

6. Toussirot E, Benmansour A, Bonneville JF, Wendling D. Atlantoaxial subluxation in an ankylosing spondylitis patient with cervical spine ossification. Br J Rheumatol 1997;36:293-5.

7. Suarez-Almazor ME, Russell AS. Anterior atlantoaxial subluxation in patients with spondyloarthropathies: association with peripheral disease. J Rheumatol 1988;15:973-5.

8. Slobodin G, Shpigelman A, Dawood H, et al. Craniocervical junction involvement in ankylosing spondylitis. Eur Spine J 2015;24:2986-90.

9. Qian BP, Mao SH, Jiang J, Wang B, Qiu Y. Mechanisms, predisposing factors, and prognosis of intraoperative vertebral subluxation during pedicle subtraction osteotomy in surgical correction of thoracolumbar kyphosis secondary to ankylosing spondylitis. Spine (Phila Pa 1976) 2017;42:E983-90.

10. Manabe N, Covaro A, Bobinski L, Shimizu T, Olerud $\mathrm{C}$, Robinson Y. The relationship between the occipitocervical junction and thoracic kyphosis in ankylosing spondylitis: a retrospective cohort study of 86 cervical fractures in surgically treated patients. Asian Spine J 2019;13:103-10.

11. Engfeldt B, Romanus R, Yden S. Histological studies of pelvo-spondylitis ossificans (ankylosing spondylitis) correlated with clinical and radiological findings. Ann Rheum Dis 1954;13:219-28.

12. Baraliakos X, Listing J, von der Recke A, Braun J. The natural course of radiographic progression in ankylosing spondylitis: differences between genders and appearance of characteristic radiographic features. Curr Rheumatol Rep 2011;13:383-7.

13. Romanus R, Yden S. Destructive and ossifying spondylitic changes in rheumatoid ankylosing spondylitis (pelvo-spondylitis ossificans). Acta Orthop Scand 1952;22:88-99.

14. Weinmann JP, Sicher H. Bone and bones: fundamentals of bone biology. St. Louis (MO): Mosby; 1955.

15. Creemers MC, Franssen MJ, van't Hof MA, Gribnau FW, van de Putte LB, van Riel PL. Assessment 
of outcome in ankylosing spondylitis: an extended radiographic scoring system. Ann Rheum Dis 2005;64:127-9.

16. Park JS, Kim J, Joo IH, Sim KD, Park YS. Analysis of risk factors for sagittal translation after pedicle subtraction osteotomy in patients with ankylosing spondylitis. Spine J 2018;18:1356-62.

17. Archer JR, Keat AC. Ankylosing spondylitis: time to focus on ankylosis. J Rheumatol 1999;26:761-4.

18. El Maghraoui A, Bensabbah R, Bahiri R, Bezza A, Guedira N, Hajjaj-Hassouni N. Cervical spine involvement in ankylosing spondylitis. Clin Rheumatol 2003;22:94-8.

19. Martel W, Holt JF, Robinson WD. A late effect on the cervical spine in ankylosing spondylitis. Ann Rheum Dis 1962;21:199-201.

20. Martel W, Holt JF, Cassidy JT. Roentgenologic manifestations of juvenile rheumatoid arthritis. Am J Roentgenol Radium Ther Nucl Med 1962;88:400-23.

21. Wilkinson M, Bywaters EG. Clinical features and course of ankylosing spondylitis; as seen in a followup of 222 hospital referred cases. Ann Rheum Dis 1958;17:209-28.
22. Abe Y, Yamada K, Abumi K, Iwasaki N, Sudo H. Long-term changes in vertebral morphology after cervical spinal fusion in adolescent pediatric patients: retrospective case series with up to a minimum 12 years of follow-up. World Neurosurg 2019;122:e76572.

23. Seo JY, Ha KY. Fate of posterior osteophytes in fused segments after anterior cervical discectomy and fusion. Spine (Phila Pa 1976) 2012;37:741-7.

24. Moon MS, Moon YW, Kim SS, Moon JL. Morphological adaptation of the bone graft and fused bodies after non-instrumented anterior interbody fusion of the lower cervical spine. J Orthop Surg (Hong Kong) 2006;14:303-9.

25. Frost HM. Biomechanical determinants of lamellar bone architecture. AAOS Instr Course Lect Ser 1969:1-21.

26. Frost HM. Mechanical determinants of bone modeling. Metab Bone Dis Relat Res 1982;4:217-29.

27. Frost HM. An approach to estimating bone and joint loads and muscle strength in living subjects and skeletal remains. Am J Hum Biol 1999;11:437-55.

28. Ha KY, Molon JN, Ahn JH, Kim YH. Fate of osteophytes and sclerosis in fused segments after lumbar fusion. Spine (Phila Pa 1976) 2014;39:E1110-5. 